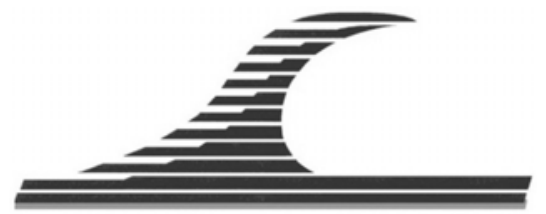

Revue Paralia, Volume 12 (2019) pp n03.1-n03.14

Mots clés : Profils de plage, Erosion, Engraissement, Transit

sédimentaire, Baie de Port-Bouët, Côte d'Ivoire.

(C) Editions Paralia CFL

\title{
Dynamique sédimentaire de la zone côtière de Port-Bouët, Côte d'Ivoire
}

\author{
Jacques André TIEMELE ${ }^{1}$, Jean-Baptiste KASSI ${ }^{1}$, \\ Jeanne Maffoué KOUADIO ${ }^{1}$
}

1. Centre Universitaire de Recherche et d'Application en Télédétection Université Félix Houphouët-Boigny de Cocody-Abidjan, Côte d'Ivoire jatiemele@yahoo.fr ; jandre.tiemele@curat-edu.org

\section{Résumé :}

Le suivi morpho-sédimentaire des côtes, effectué, à travers des mesures de levés topographiques au DGPS à haute résolution spatiale $(5 \mathrm{~cm}$ de précision) a permis d'identifier 7 profils de plage et de quantifier les changements morphologiques sur cette plage entre octobre 2014 et février 2016. Les profils de plage ont été identifiés à partir du profil topographique de la première mission de terrain (29 décembre 2014). Il s'en est suivi une interpolation de lignes 3D sur les modèles surfaciques (TIN), calculés à partir du logiciel ARCGIS 10.2.2. Les évolutions sédimentaires enregistrées sur ces profils ont montré un équilibre sédimentaire dans le secteur du Phare $\left( \pm 16000 \mathrm{~m}^{3}\right)$, suivi de sédiments engraissés entre le Phare et l'Aéroport (compris entre +3350 et +48880 $\mathrm{m}^{3}$ ), dont certaines quantités ont été érodées entre la localité de Derrière Wharf et l'Aéroport $\left(-1050 \mathrm{~m}^{3}\right)$. Ces évolutions traduisent l'instabilité du trait de côte et confirment le sens du transit sédimentaire de la dérive littorale de l'ouest vers l'est de la côte. Les quantités érodées pourraient s'expliquer par le piégeage des sédiments dans la baie de Port-Bouët dû aux effets conjugués des protections du canal de Vridi et du Trousans-fond qui entaillent l'avant-côte. 


\section{Introduction}

L'hydrodynamique littorale est le résultat d'interactions complexes entre états de mer, courants et niveaux d'eau. La compréhension de ces phénomènes hydrodynamiques est par ailleurs nécessaire à la gestion des zones côtières du fait de leur rôle dans le transport sédimentaire. Depuis les premières études sur les impacts des changements climatiques, il est apparu clairement que ces changements climatiques auront des conséquences considérables sur les zones côtières, surtout sur les petites îles (IBE \& AWOSIKA, 1991 ; ALUSA \& OGALO, 1992 ; HOOZEMANS et al., 1993 ; IBE et OJO, 1994). Ceci est dû en premier lieu à leur morphologie (côtes basses avec de nombreux estuaires et deltas), à la présence des écosystèmes importants très sensibles aux paramètres climatiques et à la forte concentration des populations et des activités économiques.

En Côte d'Ivoire, le littoral a fait l'objet de nombreux travaux de recherches montrant le caractère érosif des côtes (ABE et al., 2014). En effet, des études effectuées sur les côtes de Port-Bouët et de Jaqueville ont permis de mettre en évidence des secteurs morpho-sédimentaires distincts (N'DOUFOU et al., 2015) avec un déficit sédimentaire estimé à $2500 \mathrm{~m}^{3}$ à Port-Bouët et un bilan sédimentaire positif de $+2300 \mathrm{~m}^{3}$ à Jaqueville. Dans le secteur de San-Pedro qui abrite le deuxième Port du pays, l'analyse des levés topographiques et sédimentologiques a montré que les vitesses moyennes de recul du rivage se situent entre 1,2 à $1,6 \mathrm{~m} / \mathrm{an}$ à l'est immédiat des structures de piégeage des sédiments contre une avancée spectaculaire d'environ $8 \mathrm{~m} /$ an dans la zone du Tombolo (YAO et al., 2010). De plus, les modifications locales liées aux saisons hydrologiques ont permis de comparer les états extrêmes des profils de plage entre 1986 et 2004 de Grand-Lahou, de Jaqueville, du canal de Vridi, de Port-Bouët, de Grand-Bassam, de Mondoukou et d'Assinie. Elles ont permis de suivre les mouvements du trait de côte constatés lors de la comparaison des photographies aériennes (ABÉ, 2005).

Au phare de Port-Bouët, les levés de profils de plage ont été effectués après les tempêtes de juillet 1984 et celle de mai 1986 qui ont imprimé des reculs importants du trait de côte de l'ordre de 20 à $30 \mathrm{~m}$. L'étude que nous avons effectuée porte sur la quantification du budget sédimentaire réalisée sur des profils de la plage de Port-Bouët aux moyens d'outils à haute résolution spatiale et ce, dans le but de proposer des solutions de lutte contre le déficit sédimentaire de la zone côtière de cette partie du littoral ivoirien.

\section{Zone d'étude}

La zone d'étude concerne la côte de Port-Bouët et se situe entre $392500 \mathrm{~W}$ et $398500 \mathrm{~W}$ et entre $579200 \mathrm{~N}$ et $581600 \mathrm{~N}$ (coordonnées en mètres, projection UTM Nord, fuseau 30, voir figure 1). Elle est interrompue par le Canal de Vridi qui relie la lagune Ebrié à l'Océan Atlantique. Sa partie Est est représentée par les secteurs du Phare, du quartier 
Derrière Wharf et de l'Aéroport international Félix Houphouët Boigny (FHB). C'est un littoral bas, rectiligne, formé par une plage sableuse adossée à des cordons littoraux de grandes extensions. La plage du littoral Est de Port-Bouët est constituée de sables dont les caractéristiques mettent en évidence le tri opéré par les déferlements successifs des vagues. C'est une plage de sables essentiellement grossiers présentant une certaine homogénéité et dont les sédiments qui la constituent ont une taille médiane entre 540 et $783 \mu \mathrm{m}$.

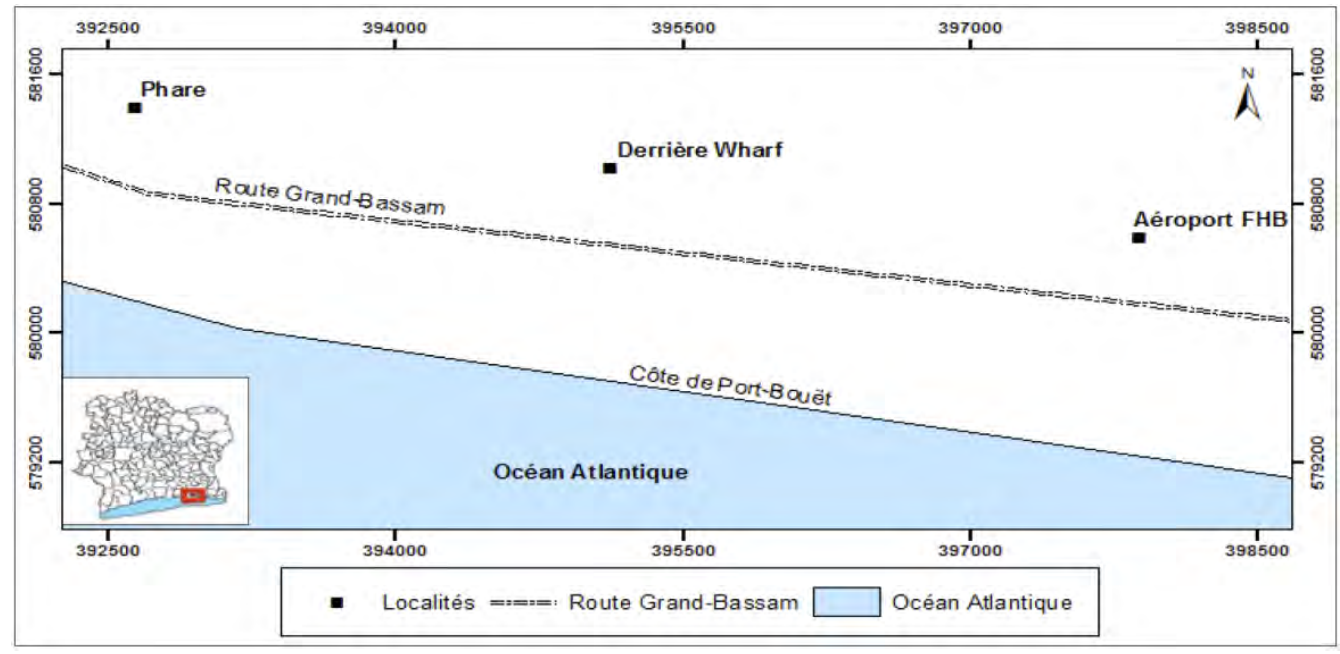

Figure 1. Présentation de la zone d'étude.

Les coordonnées sont données en mètres, projection UTM Nord, fuseau 30.

\section{Matériel et méthodes}

\subsection{Techniques d'identification des profils de plage}

Les profils de plage sont identifiés à partir des profils topographiques de la plage de Port-Bouët. Les profils topographiques quant à eux, ont été identifiés par interpolation de lignes 3D sur les modèles surfaciques (TIN) de ARCGIS 10.2.2 de la plage en date du 29 décembre 2014. Ces positions ont permis de relever les coordonnées géographiques des hauts estrans et des bas estrans de profils qui sont restées invariantes au cours des missions de terrain et de suivre l'évolution de ces profils à chaque campagne de mesures (figure 2).

\subsection{Calcul de dénivelées d'altitude}

Chaque profil de plage est déterminé par interpolation de lignes 3D reliant les points du haut estran et les points du bas estran des modèles de surface TIN. Pour chaque profil, le calcul des dénivelées d'altitude $Z$ s'obtient en soustrayant les altitudes des points invariants $Z_{o}=34,405 \mathrm{~m}$ aux altitudes mesurées $Z_{i}$ de la station fixe du DGPS, soit 
$Z=Z_{i}-Z_{o}$. Les dénivelées d'altitude $Z$ calculées, exprimées en $\mathrm{cm}$ représentent l'axe vertical du profil. L'axe horizontal, exprimé en m, représente la longueur du profil.

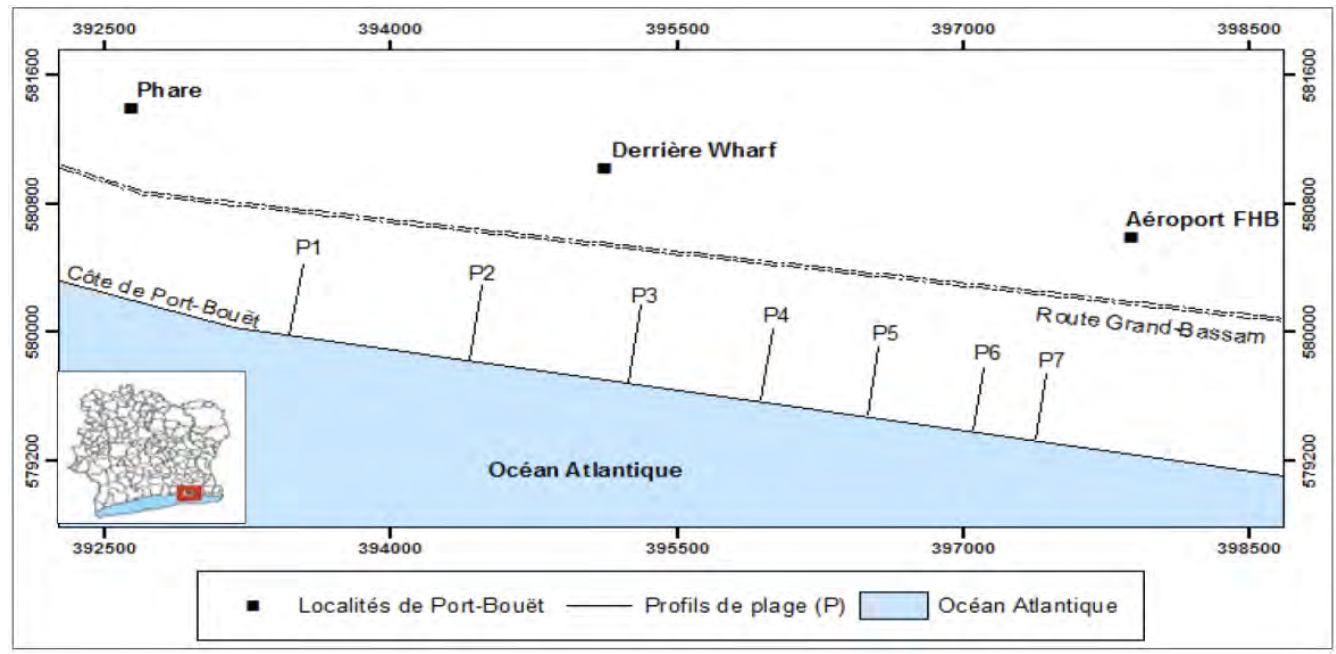

Figure 2. Dispositions des profils de plage de la zone d'étude.

Les coordonnées sont données en mètres, projection UTM Nord, fuseau 30.

\subsection{Estimation de 1'évolution sédimentaire sur les profils de plage}

Le calcul du volume de sédiments se fait en considérant sur le profil, la différence de dénivelées d'altitude sur la portion de la longueur du profil qui subit des changements morphologiques et la distance séparant deux profils. Les quantités de sédiments sont celles d'une érosion quand le volume $V_{\text {sed }}$ est négatif et celles d'un engraissement quand le calcul donne un résultat positif selon l'équation (1) suivante :

$V_{\text {sed }}=\Delta Z \times \Delta L \times D$

où : $\Delta Z$ représente les dénivelées entre deux dates, $\Delta L$ la différence de longueur sur le profil et $D$ la distance séparant chaque profil $\mathrm{P}_{\mathrm{i}}$ du profil précédent $\mathrm{P}_{\mathrm{i}-1}$. Le tableau 1 donne la distance caractéristique $\mathrm{D}$ retenue pour chaque profil.

Tableau 1. Distance D caractéristique de chaque profil.

\begin{tabular}{ll}
\hline Profil & Distance D (m) \\
\hline P1 & 200,00 \\
P2 & 480,00 \\
P3 & 520,00 \\
P4 & 200,00 \\
P5 & 100,00 \\
P6 & 200,00 \\
P7 & 100,00 \\
\hline
\end{tabular}




\section{Résultats}

4.1 Variation morpho-sédimentaire sur le profil P1

Initialement rectiligne sur $30 \mathrm{~m}$ de longueur en décembre 2014, le profil tend à s'équilibrer au 30 avril 2015. Une augmentation d'altitude sur $10 \mathrm{~m}$ du haut estran alterne avec une baisse de $150 \mathrm{~cm}$ d'altitude sur le bas estran. Le profil étant situé à $200 \mathrm{~m}$ du début de plage concerné, la quantité engraissée sur le haut estran est évaluée à $+3000 \mathrm{~m}^{3}$. Les $20 \mathrm{~m}$ de plage qui suivent sont érodés et le volume correspondant à cette érosion est estimé à $-6000 \mathrm{~m}^{3}$. Au 12 novembre 2015, les sédiments s'accumulent sur le profil et les altitudes augmentent pour atteindre une dénivelée de $600 \mathrm{~cm}$ d'épaisseur sur les premiers $8 \mathrm{~m}$ du haut estran. Elles diminuent fortement ensuite jusqu'à $350 \mathrm{~cm}$ et restent constantes entre 10 et $22 \mathrm{~m}$ de longueur du profil. L'engraissement constaté est estimé à $+4000 \mathrm{~m}^{3}$ sur les 8 premiers $\mathrm{m}$ et à $+9000 \mathrm{~m}^{3}$ sur les $18 \mathrm{~m}$ suivants. Entre le 12 novembre 2015 et le 27 février 2016, le profil devient convexe et des quantités de sédiments s'érodent sur l'ensemble des 20 mètres restant, évaluées à $-10000 \mathrm{~m}^{3}$. La variation sédimentaire sur ce profil est un équilibre de $\pm 16000 \mathrm{~m}^{3}$ (figure 3).

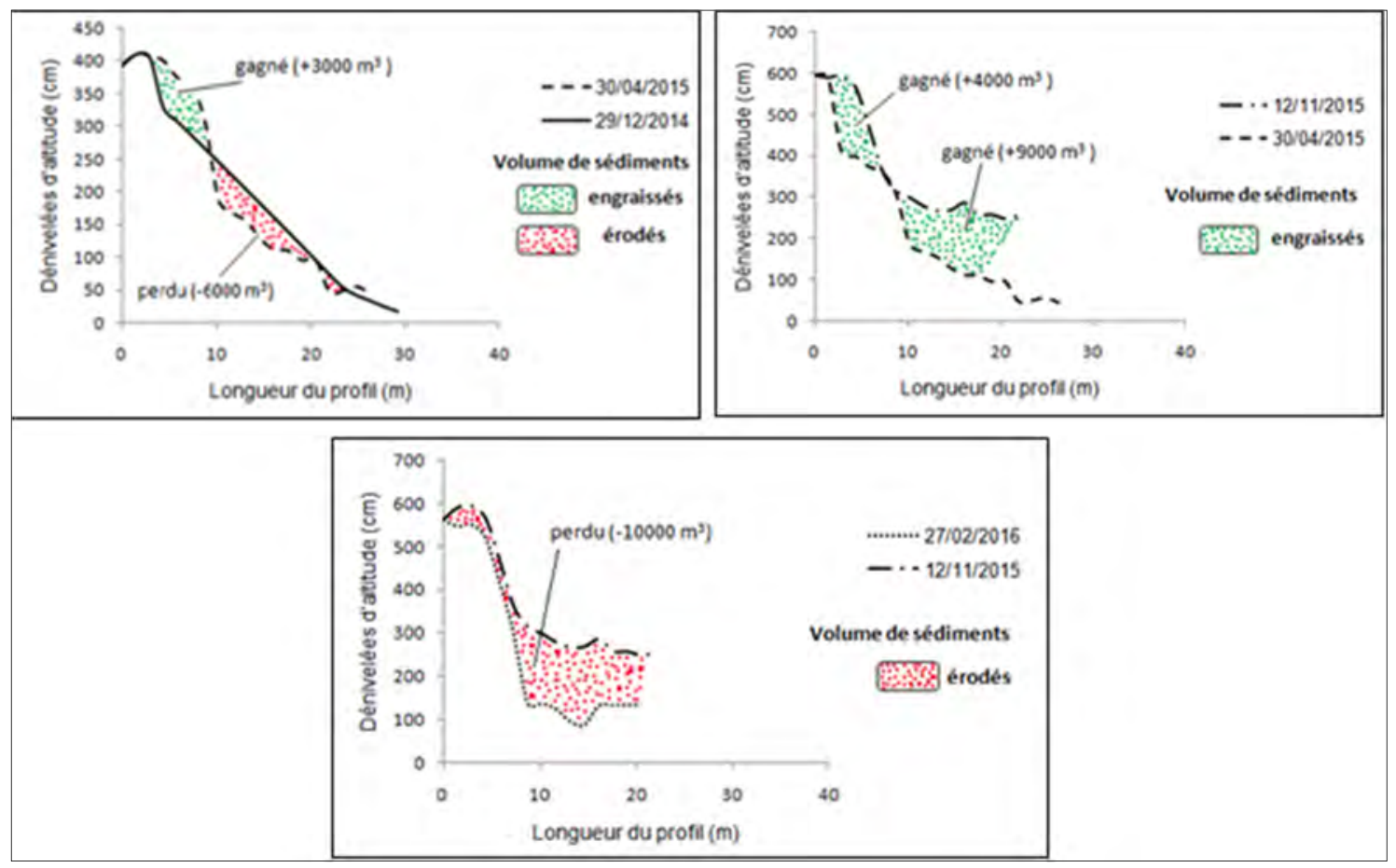

Figure 3. Evolution sédimentaire sur le profil P1. 


\subsection{Variation morpho-sédimentaire sur le profil P2}

Entre le 29 décembre 2014 et le 30 avril 2015, le profil est convexe sur $40 \mathrm{~m}$ de longueur et les sédiments s'accumulent sur toute la longueur du profil. Ce profil étant situé à $480 \mathrm{~m}$ du profil précédent, le volume de sédiments engraissés est évalué à $+10800 \mathrm{~m}^{3}$. Au 12 novembre 2015, le profil reste convexe et on assiste à une accumulation de sédiments sur $18 \mathrm{~m}$ du haut estran et sur $16 \mathrm{~m}$ du bas estran. Ces quantités accumulées sont alternées par une un volume érodé entre 18 et $23 \mathrm{~m}$ du profil sur 350 et $400 \mathrm{~cm}$ d'épaisseur. Ceci permet d'évaluer ce volume érodé à $-1200 \mathrm{~m}^{3}$ contre +21600 et $+18000 \mathrm{~m}^{3}$ de sédiments engraissés au cours cette période. Au 27 février 2016, le profil devient concave et un volume important de sédiments est érodé sur $200 \mathrm{~cm}$ d'épaisseur et évalué à $-38400 \mathrm{~m}^{3}$. L'évolution sédimentaire sur le profil P2 est estimée à $+10800 \mathrm{~m}^{3}$ (figure 4).

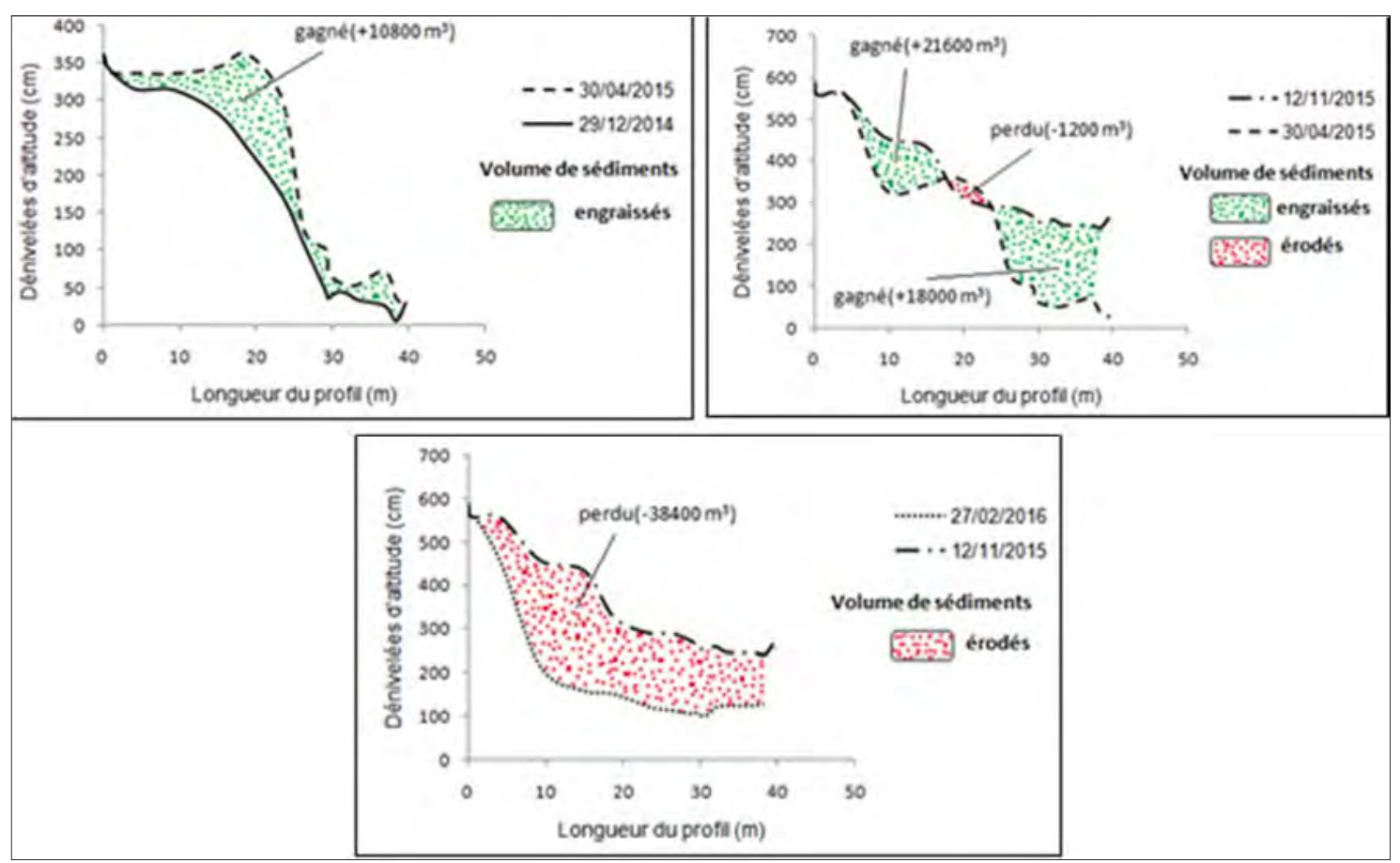

Figure 4. Evolution sédimentaire sur le profil P2. 


\subsection{Variation morpho-sédimentaire sur le profil P3}

D'une longueur de $38 \mathrm{~m}$ entre le 29 décembre 2014 et le 30 avril 2015, le profil est convexe et les altitudes augmentent entre 20 et $38 \mathrm{~m}$ du profil sur $350 \mathrm{~cm}$ au bas estran. Le profil est situé à $520 \mathrm{~m}$ du profil précédent $\mathrm{P} 2$ et il en résulte un volume sédimentaire de $+32760 \mathrm{~m}^{3}$. Cet engraissement est précédé d'une quantité érodée de $20 \mathrm{~cm}$ sur $5 \mathrm{~m}$ en haut estran. Cette quantité érodée avoisine $-520 \mathrm{~m}^{3}$ de sédiments. Entre le 30 avril 2015 et le 12 novembre 2015, le profil reste convexe et la différence d'altitude atteint $450 \mathrm{~cm}$ sur le haut estran et diminue brutalement jusqu'à $20 \mathrm{~m}$ du profil. Un volume érodé est constaté sur une dénivelée de $180 \mathrm{~cm}$ entre 25 et $30 \mathrm{~m}$ sur le profil. Pendant ce temps, l'accumulation des sédiments s'accroit sur le reste du profil. Cette période se caractérise donc par des engraissements de $+30420 \mathrm{~m}^{3}$ et de $+6240 \mathrm{~m}^{3}$ et une érosion de $-6240 \mathrm{~m}^{3}$. En février 2016, le profil reste toujours convexe et les quantités érodées surviennent sur les $5 \mathrm{~m}$ en haut estran et $15 \mathrm{~m}$ en bas estran, respectivement sur 200 et $150 \mathrm{~cm}$ d'épaisseur. Les volumes érodés sont évalués alors à $-5200 \mathrm{~m}^{3}$ et à $-11700 \mathrm{~m}^{3}$. Entre ces zones érodées, un engraissement est constaté sur $40 \mathrm{~cm}$ entre 5 et $22 \mathrm{~m} \mathrm{du}$ profil. Cette quantité engraissée avoisine $+3120 \mathrm{~m}^{3}$ de sédiments. La variation sédimentaire sur ce profil est de $+48880 \mathrm{~m}^{3}$ (figure 5).

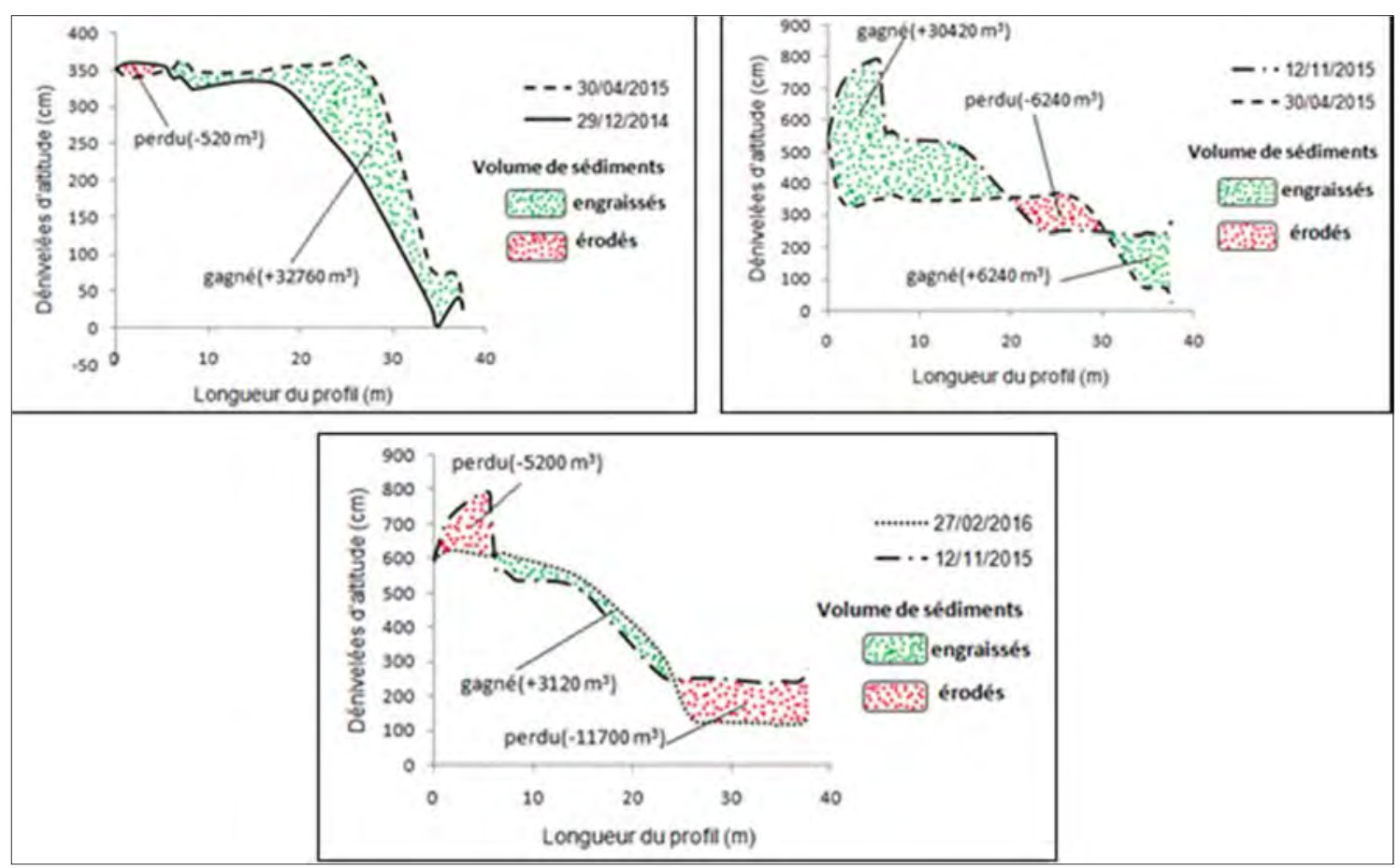

Figure 5. Evolution sédimentaire sur le profil P3. 


\subsection{Variation morpho-sédimentaire sur le profil P4}

Entre le 29 décembre 2014 et le 30 avril 2015, le profil P4 de 38 m de longueur est convexe. Il est situé à $200 \mathrm{~m}$ du profil précédent P3. Il subit une érosion de $160 \mathrm{~m}^{3}$ sur le haut estran et un engraissement d'une épaisseur de $150 \mathrm{~cm}$ entre 8 et $32 \mathrm{~m}$ le 30 avril 2015 , estimé à $+7500 \mathrm{~m}^{3}$. Au 12 novembre 2015, le profil reste convexe et les sédiments s'accumulent sur une épaisseur moyenne de $200 \mathrm{~cm}$ sur le profil, ce qui porte à $+15200 \mathrm{~m}^{3}$ la quantité de sédiments engraissés. Au 27 février 2016, le profil prend une allure concave et les sédiments sont érodés. Cette érosion survient sur une dénivelée moyenne de $200 \mathrm{~cm}$. Le volume érodé est de $-15200 \mathrm{~m}^{3}$. L'évolution sédimentaire sur ce profil est de $+7340 \mathrm{~m}^{3}$ (figure 6).

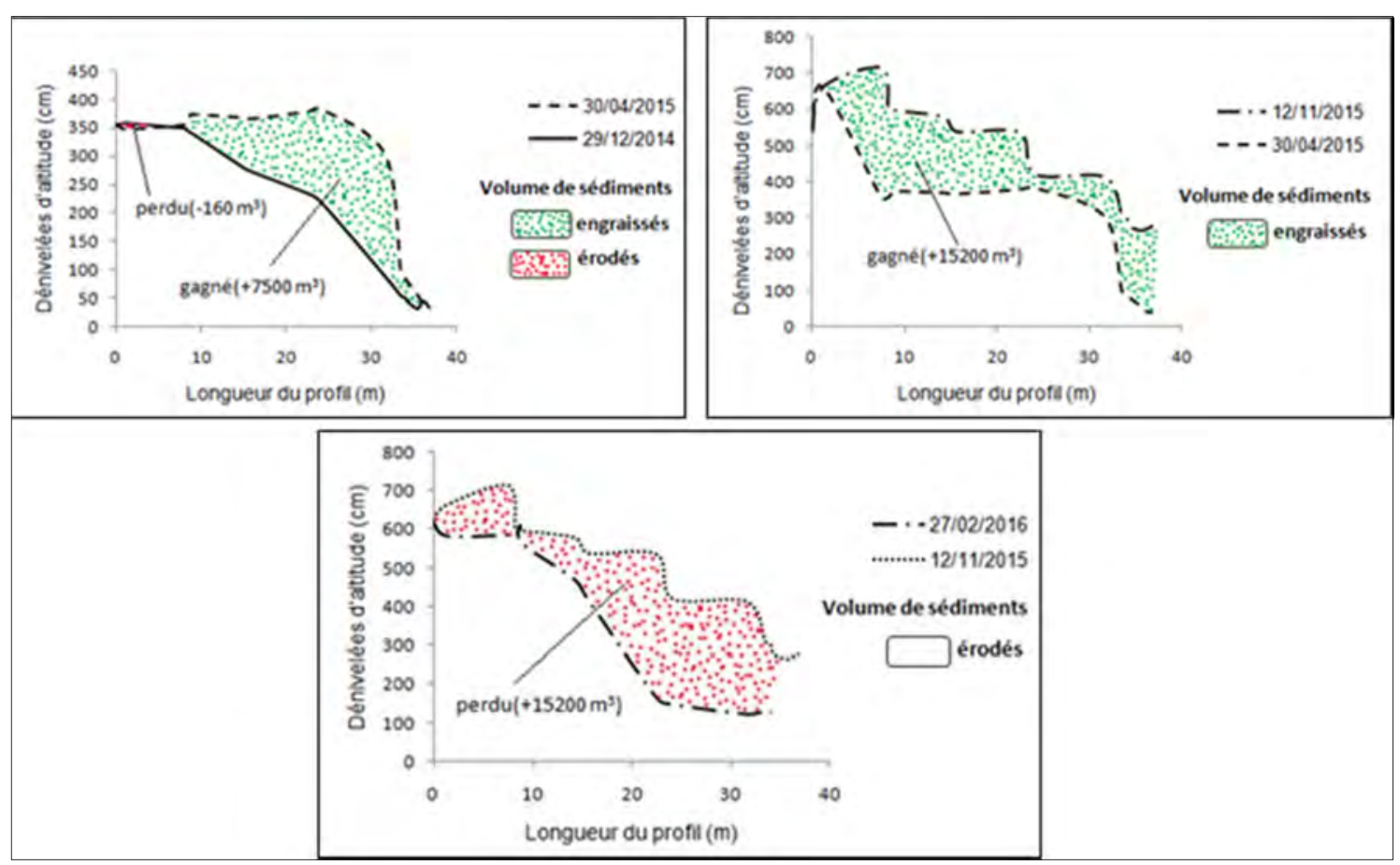

Figure 6. Evolution sédimentaire sur le profil P4. 


\subsection{Variation morpho-sédimentaire sur le profil P5}

D'une allure convexe au 29 décembre 2014, les changements morphologiques sur ce profil montrent au 30 avril 2015, une pente abrupte à partir de $10 \mathrm{~m}$ en haut estran sur $350 \mathrm{~cm}$ d'épaisseur. C'est un profil de $38 \mathrm{~m}$ situé à $100 \mathrm{~m}$ du profil $\mathrm{P} 4$. Les sédiments sont érodés avec un volume estimé à $-9800 \mathrm{~m}^{3}$. Au 12 novembre 2015, le profil reste convexe avec une longueur réduite à $35 \mathrm{~m}$. Les sédiments s'accumulent sur $250 \mathrm{~cm}$ d'épaisseur. Le volume de sédiments engraissés avoisine $+8750 \mathrm{~m}^{3}$. Au 27 février 2016, l'allure convexe du profil n'a pas provoqué de changement morphologique. Il n'y a pas eu de variation sédimentaire au cours de cette période. La variation sédimentaire sur ce profil est évaluée à -1050 $\mathrm{m}^{3}$ (figure 7).

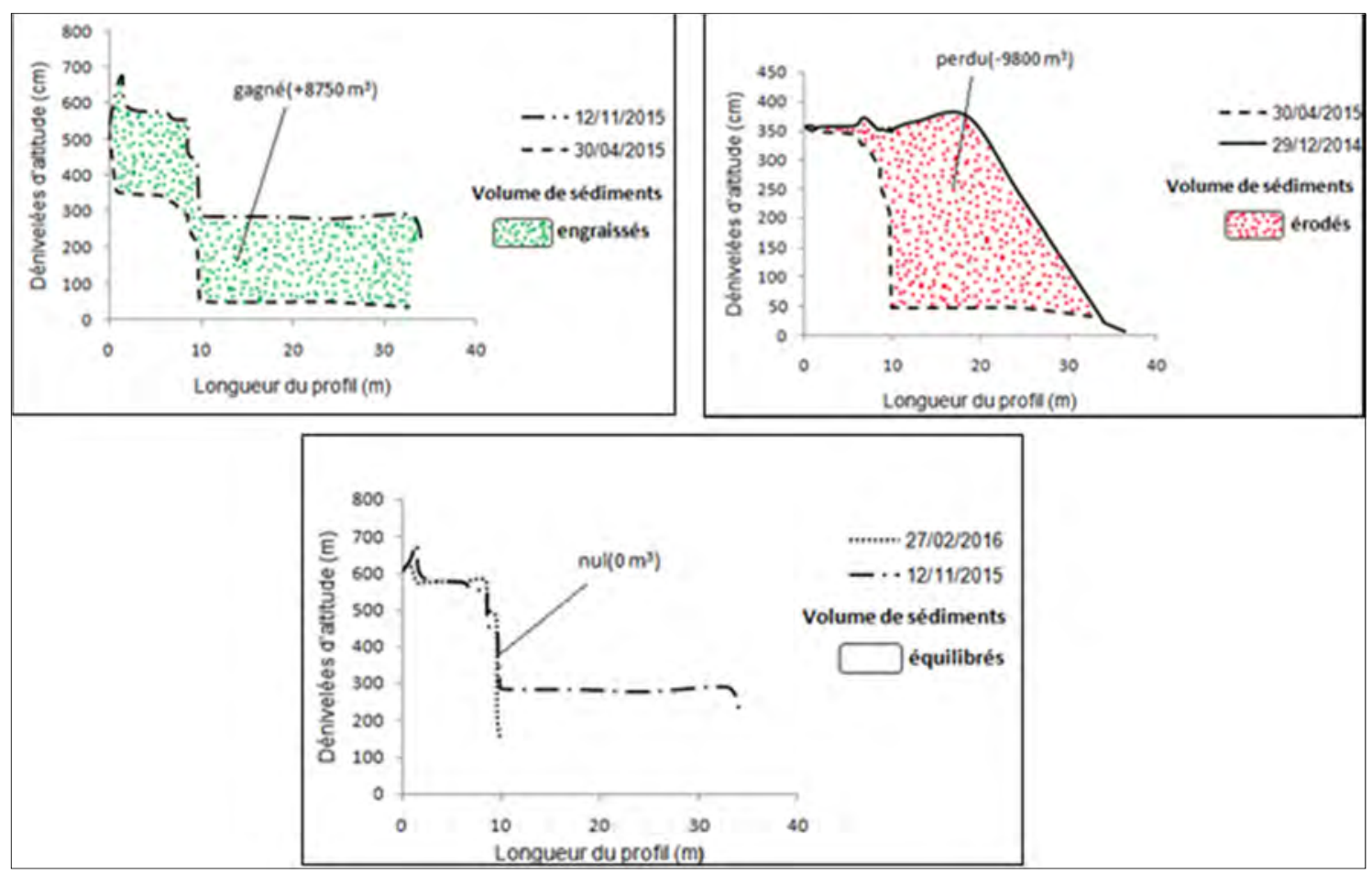

Figure 7. Evolution sédimentaire sur le profil P5. 


\subsection{Variation morpho-sédimentaire sur le profil P6}

Entre le 29 décembre 2014 et le 30 avril 2015, le profil a une longueur de $32 \mathrm{~m}$ et une allure convexe. Une accumulation de sédiments survient sur $150 \mathrm{~cm}$ d'épaisseur en moyenne. Le profil se situe à $200 \mathrm{~m}$ du profil P5 et le volume de sédiments engraissés est de $+6600 \mathrm{~m}^{3}$. Au 12 novembre 2015, le profil reste convexe mais se rétrécit de $10 \mathrm{~m}$. Il subit une hausse de $2 \mathrm{~m}$ d'altitude sur les $7 \mathrm{~m}$ du haut estran avec un engraissement de sédiments. Cet engraissement est suivi d'une érosion de $1 \mathrm{~m}$ entre 7 et $23 \mathrm{~m} \mathrm{du}$ profil. Les quantités de sédiments engraissés et érodés sont respectivement évaluées à $+2800 \mathrm{~m}^{3}$ et $-3200 \mathrm{~m}^{3}$. Au 27 février 2016, le profil prend une allure concave et se rallonge à nouveau pour atteindre $32 \mathrm{~m}$. Il est marqué par une accumulation de sédiments sur $22 \mathrm{~m}$ de longueur et sur $1 \mathrm{~m}$ de dénivelée d'altitude. Une quantité $+4400 \mathrm{~m}^{3}$ de sédiments est alors accumulée au cours de cette période contribuant ainsi à une variation sédimentaire estimée à $+10600 \mathrm{~m}^{3}$ sur le profil (figure 8).

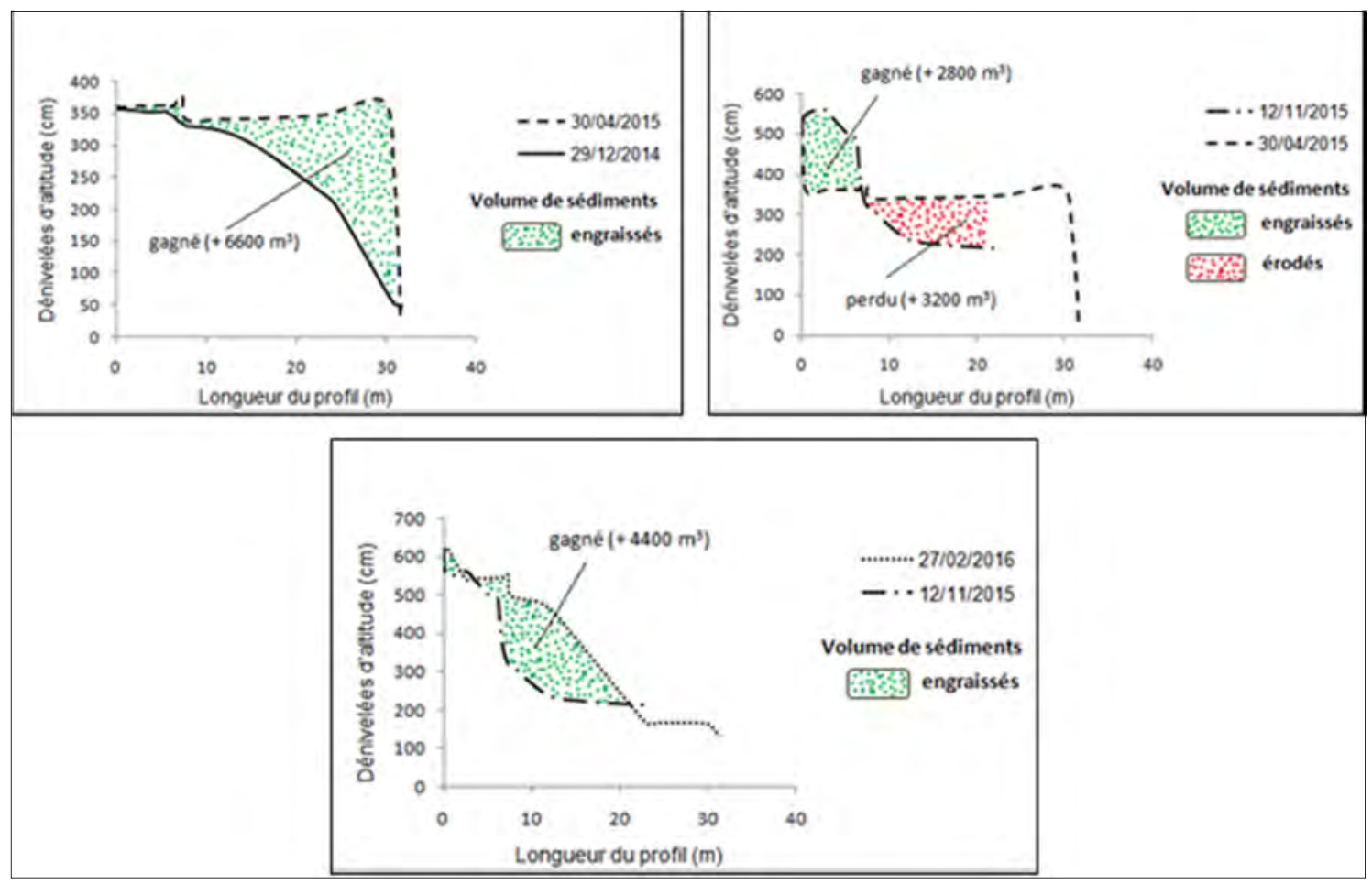

Figure 8. Evolution sédimentaire sur le profil P6. 


\subsection{Variation morpho-sédimentaire sur le profil P7}

Le profil est situé à $100 \mathrm{~m}$ du profil précédent P6 et long de $30 \mathrm{~m}$ entre le 29 décembre 2014 et le 30 avril 2015. Il a une allure convexe caractérisée par un engraissement de sédiments sur $1 \mathrm{~m}$ d'altitude et sur $22 \mathrm{~m}$ du profil. Cet engraissement est suivi d'une érosion de $50 \mathrm{~cm}$ entre 22 et $28 \mathrm{~m}$ du profil. Ceci porte les variations sédimentaires respectivement à $+2200 \mathrm{~m}^{3}$ et à $-300 \mathrm{~m}^{3}$ au cours de cette période. Au 12 novembre 2015, l'allure du profil reste convexe. Des quantités de sédiments s'engraissent sur les $15 \mathrm{~m}$ du haut estran et entre 22 au $28 \mathrm{~m}$ du bas estran. Un volume érodé de sédiments survient entre 15 et $20 \mathrm{~m}$ du profil. Cette période se caractérise donc par des quantités accumulées de sédiments de $+2250 \mathrm{~m}^{3}$ et $+900 \mathrm{~m}^{3}$ entre lesquelles le volume érodé a été évalué à $-500 \mathrm{~m}^{3}$. En février 2016, le profil devient concave et sa longueur se réduit de $6 \mathrm{~m}$. Une érosion de $1 \mathrm{~m}$ est constatée entre 5 et $22 \mathrm{~m}$ de la longueur du profil, soit $1700 \mathrm{~m}^{3}$ de sédiments érodés. Cette érosion est précédée d'un engraissement de $1 \mathrm{~m}$ sur les $5 \mathrm{~m}$ du haut estran, soit $+500 \mathrm{~m}^{3}$ de sédiments engraissés. L'évaluation sédimentaire sur ce profil conduit donc à un engraissement de $+3350 \mathrm{~m}^{3}$ de sédiments (figure 9).

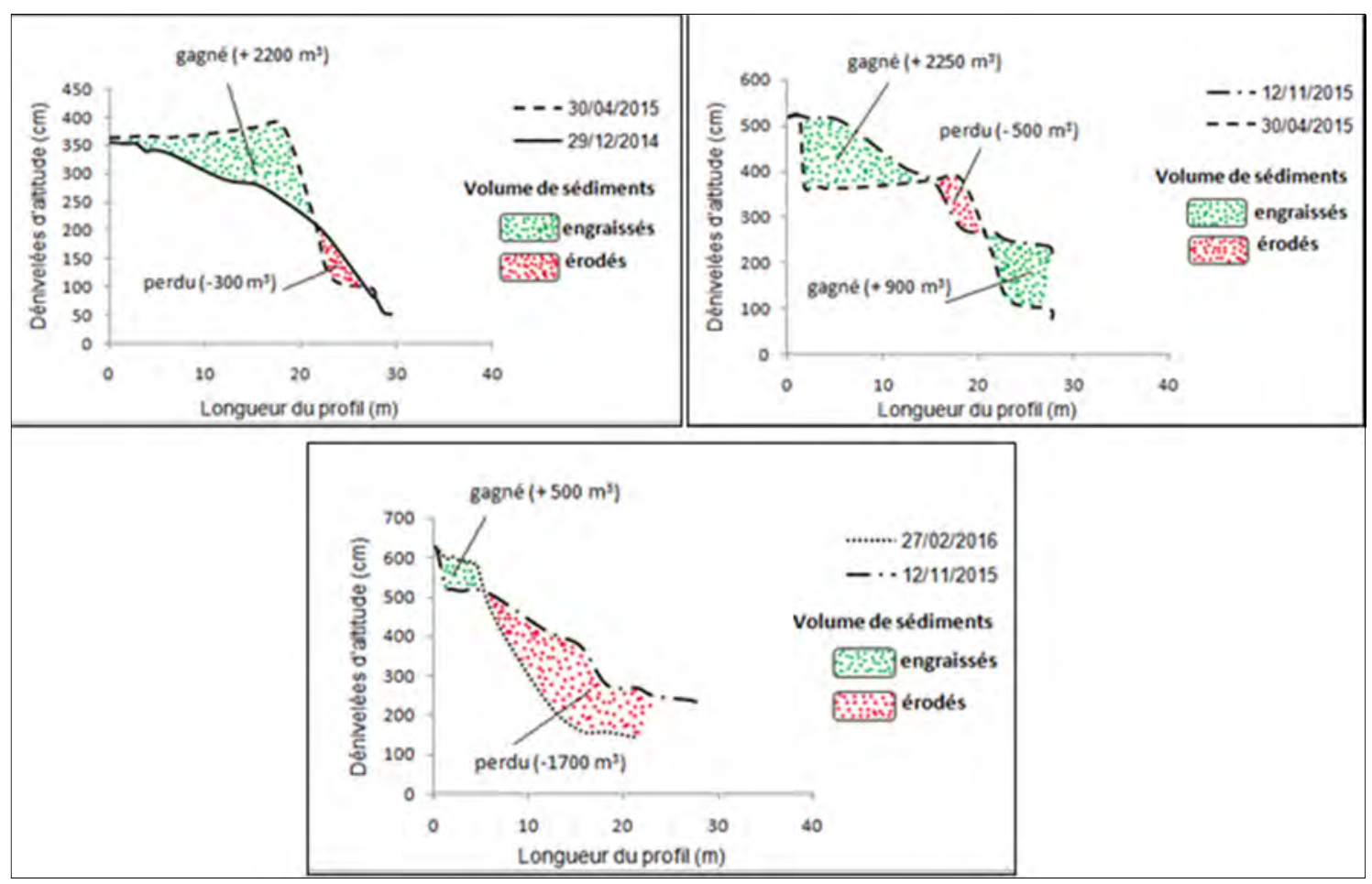

Figure 9. Evolution sédimentaire sur le profil P7.

4.8 Dynamique sédimentaire observée sur les profils de plage de Port- Bouët

La réalisation des évolutions sédimentaires sur les profils de plage a permis de présenter la dynamique sédimentaire des secteurs de la côte de Port-Bouët entre le 29 décembre 2014 et le 27 février 2016. Les profils P2, P3, P4, P6 et P7 ont des variations sédimentaires positives. Elles traduisent une recharge sédimentaire dans les secteurs de 
Derrière Wharf et de l'Aéroport FHB. Le profil P5 quant à lui, présente une variation sédimentaire négative. Les sédiments sont alors érodés en direction des secteurs de la côte entre la localité de Derrière Wharf et l'Aéroport. Seul le profil P1 se caractérise par une évolution sédimentaire nulle, c'est-à-dire qu'il y a eu autant de sédiments engraissés et érodés. Il n'y a donc pas eu de variation morphologique dans le secteur de Derrière Wharf (figure 10).

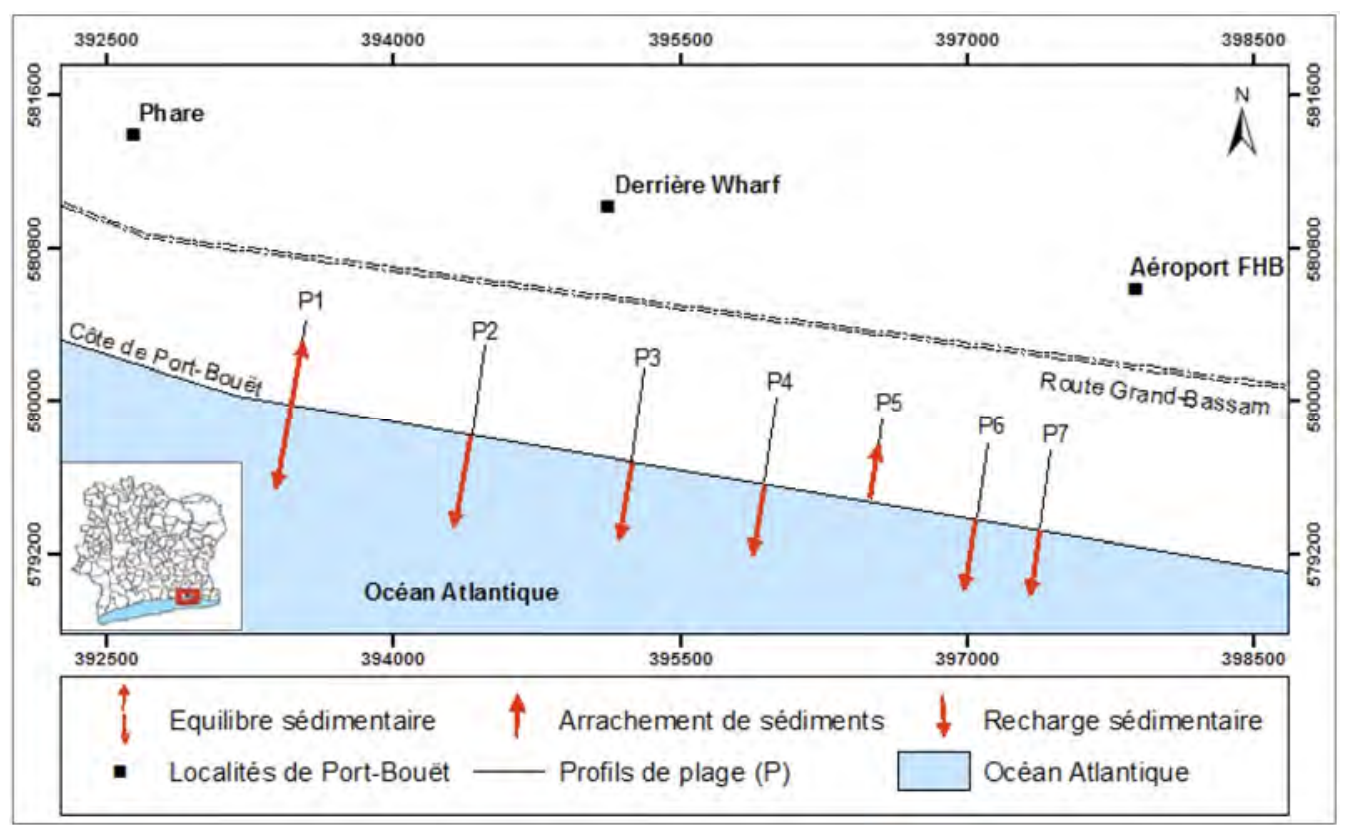

Figure 10. Dynamique sédimentaire sur les profils de plage Port-Bouët entre décembre 2014 et février 2016.

\section{Discussion}

Les tendances morpho-sédimentaires évolutives sur les profils de plage de Port-Bouët permettent de dresser un bilan sédimentaire positif sur cette zone côtière. En effet, le secteur du Phare apparemment stable, s'engraisse peu à peu le long de la côte en direction de l'Aéroport FHB. La théorie de la dérive littorale qui évoque le transit sédimentaire de l'ouest vers l'est de la côte confirme ici les résultats des changements morphologiques sur ces profils. Cependant des épisodes d'érosion se traduisant par des arrachements de sédiments entre la localité de Derrière Wharf et l'Aéroport sont enregistrés. Cela vient du fait que, dans ce secteur, la quantité érodée l'emporte sur le volume de sédiments engraissés. Ceci est confirmé par les travaux des auteurs (TASTET, 1987 ; IBE, 1988 ; BLIVI, 1993 ; PASKOFF, 1993 ; ABE et BAKAYOKO, 1995 ; DIBAJNIA et al., 2004 in YAO, 2012), qui estiment que l'interruption du transport longitudinal des sédiments est causée par la présence d'infrastructures côtières et provoque une extension des plages situées en amont tandis que les plages en aval s'érodent. De plus, à l'est de Port-Bouët, TASTET (1987) a évalué la capacité de 
transport des sédiments de la dérive littorale à $400000 \mathrm{~m}^{3} / \mathrm{an}$. En effet, les houles qui abordent la côte régulièrement avec un angle oblique, sont à l'origine d'un important transit sédimentaire. Cette capacité est différente du volume sédimentaire évalué à PortBouët au cours de nos travaux. En clair, les profils 6 et 7 situés dans le secteur côtier de l'Aéroport montrent que les évolutions sédimentaires sont estimées respectivement à +10600 et $+3300 \mathrm{~m}^{3}$, soit $13900 \mathrm{~m}^{3}$ de sédiments entre 2014 et 2016 ou $7000 \mathrm{~m}^{3} / \mathrm{an}$. Ces différences pourraient s'expliquer d'une part par des courants d'arrachement entre les secteurs de Derrière Wharf et de l'Aéroport FHB qui provoquent le transport sédimentaire dans le sens contraire du courant de dérive, c'est-à-dire de l'est vers l'ouest, et d'autre part, par le piégeage des sédiments dans la baie de Port-Bouët à cause des effets conjugués des protections du canal de Vridi et du Trou-sans-fond qui entaillent l'avant-côte. Il en résulte un déficit de la plage du littoral Est de Port-Bouët qui apparaît comme la "tête de pont" d'une nouvelle dérive sous saturée et mobilise les sédiments en direction de Grand-Bassam.

\section{Conclusion}

En définitive, il convient de retenir que les mesures de levés topographiques effectuées au DGPS ont permis de suivre avec précision, la dynamique sédimentaire sur la côte de Port-Bouët entre octobre 2014 et février 2016. Les tendances ont montré des évolutions sédimentaires nulle, négative et positive, respectivement à $200 \mathrm{~m}$ du Phare (profil P1), à $1200 \mathrm{~m}$ du Phare (profil P5) et sur les autres profils de plage. Elles ont donc permis de dresser un bilan sédimentaire positif sur cette plage. Des campagnes de prélèvement et d'études sédimentologiques du bas et du haut estran de cette zone côtière devront être envisagées afin de modéliser la dispersion spatiale des sédiments sur ces côtes.

\section{Références bibliographiques}

ABE J. (2005). Contribution à la connaissance de la morphologie et de la dynamique sédimentaire du littoral ivoirien (cas du littoral d'Abidjan), essais de modélisation en vue d'une gestion rationnelle. Thèse de doctorat d'état ès-sciences naturelles, spécialité océanologie, Université de Cocody, 353 p.

ABE J., BAKAYOKO S. (1995). Influence des structures de protection d'un canal portuaire sur la morphologie de la côte : le canal de Vridi en Côte d'Ivoire. Coastal Systems and Sustainable Development in Africa. In UNESCO Reports in Marine Sciences, Vol. 66, pp 25-33.

ABE J., N'DOUFOU G.H.C., KONAN K.E, YAO K.S., BAMBA S.B. (2014). Relations entre les points critiques d'érosion et le transit littoral en Côte d'Ivoire. Africa Geoscience Review, Vol. 21, pp 1-2.

ALUSA A.L., OGALO L.T. (1992). Implication of expected climate change in the Eastern African Coastal region, UNEP, Nairobi. 
BLIVI A. (1993). Géomorphologie et dynamique actuelle du littoral du Golfe du Bénin (Afrique de l'Ouest). Thèse en géologie, Université Michel de Montaigne de Bordeaux 3, $458 \mathrm{p}$.

DIBAJNIA M., NAIRN R.B., ROSS R. (2004). Analysis of long-term sand accumulation at a harbor using $2 \mathrm{DH}$ numerical simulation. Coastal Engineering, Vol 51, pp 863-882. https://doi.org/10.1016/j.coastaleng.2004.07.013

HOOZEMANS F.M.J., MARCHAND M., PENNEKAMP H.A. (1993). Sea level rise: A global vulnerability assessment vulnerability assessments for population, coastal wetlands and rice production on a global scale. Delft Hydraulics I., The Hague, 184 p. IBE A.C., AWOSIKA L.F. (1991). National assessment and effect of sea level rise on the Nigerian Coastal Zone. Unpublished report to the University of Maryland, $31 \mathrm{p}$. IBE A.C., OJO S.O. (1994). Implications of expected climate change in the West and Central African Region: An overview. UNEP: Nairobi. UNEP Regional Seas Reports and Studies, $\mathrm{N}^{\circ} 148$, UNEP, 1994, 43 p.

IBE A.C. (1988). Artificial structures and shorelines. Kluwer Academic Publications, Nigeria. In Walker H.J. (Ed.), Dordrecht, pp 287-294. https://doi.org/10.1007/978-94-009-2999$\underline{933}$

N'DOUFOU G., ABE J., BAMBA S., HAUHOUOT C., AKA K. (2015). Effets de l'ouverture du Canal de Vridi sur les stocks sédimentaires littoraux entre Abidjan et Jacqueville (Côte d'Ivoire). Revue Paralia, Vol. 8, pp 1.1-1.16. https://doi.org/10.5150/revue-paralia.2015.n01

PASKOFF R. (1993). Côtes en dangers. Collection pratique de la Géographie, Masson, Paris, $250 \mathrm{p}$.

TASTET J. P. (1987). Effets de l'ouverture d'un canal d'accès portuaire sur l'évolution naturelle du littoral d'Abidjan (Afrique de l'Ouest). Bull. Inst. Geol. Bassin d'Aquitaine, Bordeaux, n²41, pp 177-190.

YAO K. S. (2012). Etude de la dynamique sédimentaire du littoral occidental ivoirien entre Tabou et Sassandra : approches morpho-bathymétriques, sédimentologiques et exoscopiques. Thèse de doctorat unique, Université Félix Houphouët-Boigny de Cocody-Abidjan, $197 \mathrm{p}$.

YAO K.S., ABE J., BAMBA S.B., KONAN K.E., AKA K. (2010). Dynamique d'un périmètre littoral portuaire : la côte de San-Pédro, Sud-Ouest de la Côte d'Ivoire. Revue Paralia, Vol. 3, pp 2.1-2.12. https://doi.org/10.5150/revue-paralia.2010.002 